
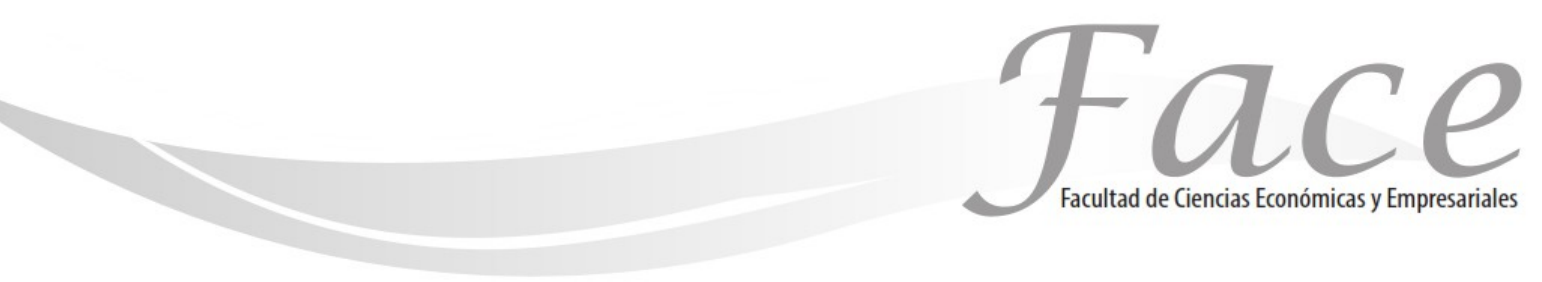

ISSN Impreso: 1794-9920

ISSN Electrónico: 2500-9338

Volumen 17- $\mathrm{N}^{\circ} 2$

Año 2017

Págs. 43 - 54

\title{
FACTORES DETERMINANTES DEL MODELO PEDAGÓGICO PRESENTES EN LOS PROYECTOS EDUCATIVOS DE PROGRAMA; ESTUDIO DE CASO DESDE LA FACULTAD DE CIENCIAS ECONÓMICAS Y ADMINISTRATIVAS DE LA UNIVERSIDAD DE NARIÑO APORTES PARA UNA RESIGNIFICACIÓN. *
}

Fabio Mejía Zambrano **

Enlace ORCID: http://orcid.org/ 0000-0002-6689-3345

Fecha de Recepción: diciembre 292016

Fecha de Aprobación: abril 72017

\section{Resumen:}

El documento presenta la importancia del modelo pedagógico en la estructura de un Proyecto Educativo de Programa-PEP- en el campo de las ciencias económicas y administrativas, referenciando un estudio de casos en la Universidad de Nariño, susceptible de ser generalizado para ser tomado como objeto de reflexión en cualquier contexto, en razón de la coherencia que debe guardar con el Proyecto Educativo Institucional-PEI. Este estudio, de corte hermenéutico, toma la teórica de la pedagogía de Flórez Ochoa, la perspectiva curricular de Carr y Kemmis, y, especialmente, los aportes de Not, De Zubiría Samper y Morín, en donde se exhorta a transcender la racionalidad normativa para apropiarse de la realidad pedagógica universitaria.

Palabras Claves: Proyecto Educativo, Modelo Pedagógico, Ciencias Económicas y Administrativas, Educación Superior.

\footnotetext{
* Profesional en Comercio Internacional y Mercadeo- Especialista en Gerencia de Mercadeo- Especialista en Negocios Internacionales-Magister en Mercadeo Agroindustrial y Docencia Universitaria. Correo electrónico: fmejiaz@ut.edu.co
} 


\title{
DETERMINANTS OF THE PEDAGOGICAL MODEL PRESENT IN THE PROGRAMME EDUCATIONAL PROJECTS; CASE STUDY FROM THE FACULTY OF ECONOMIC AND ADMINISTRATIVE SCIENCES OF THE UNIVERSITY OF NARIÑO: CONTRIBUTIONS FOR A RESIGNIFICATION.
}

\begin{abstract}
The document presents the importance of the pedagogical model in the structure of an educational project of program-PEP-in the field of economic and administrative sciences, referencing a case study at the University of Nariño, susceptible to be generalized to be taken as an object of reflection in any context, because of the coherence that should be kept with the educational project institutional-PEI. This study, from Corte Hermeneutic, it takes the theoretical of the pedagogy of Flórez Ochoa, the curricular perspective of Carr and Kemmis, and, especially, the contributions of Not, of Zubiría Samper and Morin, where it is exhorted to transcend the normative rationality to appropriate the pedagogical reality University.
\end{abstract}

Keywords: Educational Project, Pedagogical Model, Economic and Administrative Sciences, Higher Education.

\section{FACTORES DETERMINANTES DO MODELO PEDAGÓGICO PRESENTES NOS PROJECTOS EDUCATIVOS DE PROGRAMA; ESTUDO DE CASO DESDE A FACULDADE DE CIÊNCIAS ECONÓMICAS E ADMINISTRATIVAS DA UNIVERSIDADE DE NARIÑO: CONTRIBUAS PARA UMA RESIGNIFICACIÓN}

\begin{abstract}
Resumo
O artigo apresenta a importância do modelo pedagógico na estrutura de um Projecto Educativo de Programa-PEP- no campo das ciências económicas e administrativas, referindo um estudo de casos na Universidade de Nariño, susceptível de ser generalizado para ser tomado como objeto de reflexão em qualquer contexto, em razão da coerência que deve guardar como Projecto Educativo Institucional-PEl. Este estudo, de corte hermenêutico, leva a teórica da pedagogia de Flórez Ochoa, a perspectiva curricular de Carr e Kemmis, e, especialmente, as contribuições de Not, De Zubiría Samper e Morín, em onde se exhorta a transcender a racionalidade regulamento para apropriar da realidade pedagógica universitária.
\end{abstract}

Palavras-chave Projecto Educativo, Modelo Pedagógico, Ciências Económicas e Administrativas, Educação Superior. 


\section{INTRODUCCIÓN:}

El Proyecto Educativo del Programa-PEP- en coherencia con el Proyecto Educativo Institucional-PEl- debe concebirse como un documento que contiene el direccionamiento estratégico que orienta la administración curricular de un programa académico y explicitar en la fundamentación teórica, los constructos relacionados con la teoría curricular y el modelo pedagógico con sus correspondientes dimensiones.

Se indagó los rasgos de los modelos pedagógicos presentes en los PEP de los programas académicos de la Universidad de Nariño: Administración de Empresas, Contaduría Pública, Comercio Internacional, Mercadeo y Economía, partiendo del principio que se constituyen en reflexión propositiva de la Facultad de Ciencias Económicas y Administrativas como Unidad Académica, sin fronteras pedagógicas, capaces de romper las barreras que permitan reflexiones articuladoras en los procesos de enseñanza y aprendizaje.

Este estudio, con perspectiva interpretativa, está estructurado de la siguiente manera: se sintetiza los discursos de teóricos tales como currículo, pedagogía y didáctica; luego se explica el objeto de análisis, tal como se presenta en los PEP, considerando las diferentes dimensiones del modelo pedagógico, como son el epistemológico, filosófico, planificación escolar, curricular, sociológica, psicológica y evaluativa, con el fin de clasificar los hallazgos en tres modelos pedagógicos, a saber: heteroestructurante, autoestructurante e interestructurante; seguidamente se realiza una discusión a partir de la cual se derivan a manera de síntesis las conclusiones.

\section{MARCO TEÓRICO:}

2. DEBATE CONTEMPORÁNEO DE LOS MODELOS PEDAGÓGICOS: PARADIGMA, ENFOQUE, CORRIENTES Y TENDENCIAS EN UN PROYECTO EDUCATIVO DE PROGRAMA.

Morín (2003) enfatiza que el modelo pedagógico es el medio fundamental del PEI y que éstos coinciden en el desarrollo de las funciones sustantivas. Empero, dicho concepto suele ser confundido con los paradigmas, los enfoques, las corrientes, tendencias pedagógicas, modelo educativo y modelo didáctico, razón por la cual se procede a realizar la diferenciación correspondiente.

Paradigma. Parafraseando a Kuhn (1986), es un conjunto de convicciones, valores y comportamientos compartidos por los miembros de una determinada comunidad; también se caracteriza porque hace parte del inconsciente colectivo. En esencia, son un sistema de ideas catalogadas como verdaderas, capaces de sustentar su propia validez y que ha prevalecido en el tiempo. El ejemplo clásico procedente en este estudio es el constructivismo, que transitó como corriente y luego como modelo, para instaurarse a juicio de Morales (2008) como paradigma.

Enfoque. Considerando los aportes de Morales (2008), es una alternativa de entender, organizar y realizar la educación y por lo tanto una manera de abordar los procesos de enseñanza y aprendizaje, que dan origen y a la vez fundamentan a las corrientes y modelos pedagógicos. Los enfoques, desde los constructos que los sustentan, permiten realizar interpretaciones, formular criterios, lineamientos, políticas y estrategias pedagógicas.

Corriente. Es una línea de pensamiento pedagógico de connotación innovadora que se encuentra en proceso de investigación, sistematización y validación; de hecho, son tendencias fuertemente marcadas de la educación, que aún no se estructura con la jerarquía de modelo ni en la fundamentación de un enfoque, pero que progresivamente va ganando adeptos y por ende tiene la posibilidad de crear su propia escuela. (Canfux, 1996).

Tendencias. Según Morales (2008), es una perspectiva educativa que complementa, refuerza o modifica un paradigma, enfoque, modelo o corriente pedagógica. Así mismo, expresan las demandas sociales en materia de educación y genera los aportes más relevantes en las investigaciones educativas de punta y en consecuencia permiten actualizar constantemente el currículo para responder a los retos sociales y científicos.

Modelo Educativo. Cardoso (2007) cita que es un conjunto correlacionado de factores históricos, sociales, culturales y de modalidades de enseñanza y aprendizaje individual y colectivo a la vez. Es una guía que enviste de identidad a cualquier entidad de carácter educativo. Es una pretensión propositiva para generar normas institucionales que consolidadas por la comunidad universitaria explicite los elementos constitutivos del PEI.

Modelo Didáctico. Medina, Mata \& Arroyo (2002) explican que un modelo didáctico no es más que la representación de los precoces de enseñanza y aprendizaje, que facilita su conocimiento y propicia un mejoramiento continuo al seleccionar e identificar la relación de simbiosis entre ellos. Parafraseando a Joyce, Weil y Calhoun (2002) son por excelencia modelos de aprendizaje, que se dan en diferentes momentos, en la perspectiva de reconocer a la enseñanza en enseñar a como aprender.

2.1. Modelo Pedagógico, Modelo Educativo y Modelo Didáctico Not (1983 y 1992), en la historia básicamente, transversalmente, han existido dos grandes modelos 
pedagógicos: el heteroestructurante y el autoestructurante, no obstante, se debe reconocer que se han planteado diversas perspectivas teóricas que gravitan alrededor del tema; empero, emergen en hora buena, las metodologías interestructurantes, asociadas al diálogo y a la valoración del proceso de aprendizaje, (Feuerstein \& Hoffman, 1992).

Sintetizando a De Zubiría Samper (2011), explica que en los modelos heteroestructurantes el conocimiento se gesta en el entorno del aula; argumenta que la misión de la academia, en esencia, es transmitir los resultados de la cultura humana a los discentes. En este sentido, se maximiza el papel del profesor y lo considera como epicentro del proceso educativo. En cuanto a los modelos autoestructurantes, el autor precitado especifica que el estudiante en el centro del proceso educativo, en consecuencia, la educación es forjado como un proceso complejo orientado y fortalecido por una dinámica interna.

En este escenario, la academia tiene el reto de favorecer la socialización y ser la mentora de motivar el interés del estudiante. De otra parte, se enarbola el modelo dialogante, alternativa que propende por el desarrollo cognitivo, valorativo y praxiológico del estudiante, trabajando contenidos y evaluando en la trayectoria de las dimensiones humanas a partir de estrategias interestructurantes, reivindicando el rol activo del docente y estudiante (De Zubiría, 2011). Por consiguiente, implica apropiarse de la teoría de la modificabilidad cognitiva, en donde el actor convergente y el mentor del efecto Pigmalión es el profesor; así, evocando a Falik y Feurstein (1990), es preciso citar que "lo único inmodificable es la capacidad de modificar".

Ahora bien, desde el multiverso teórico de la pedagogía, se identifica que los diferentes modelos pedagógicos, desde sus particularidades, se enfocan a resolver los interrogantes: ¿qué enseñar? y ¿cómo lograr aprendizajes en los estudiantes?, coincidiendo que la cuestión fundante es satisfacer la pregunta ¿cómo enseñar lo seleccionado?

\section{-DIMENSIONES DEL MODELO PEDAGÓGICO}

En la línea de Flórez (1994) las dimensiones del modelo pedagógico, debe responder a las siguientes pregunta: desde lo epistemológico, ¿ cuál es la orientación teórica de los planes de estudio?; en la perspectiva filosófica, ¿qué tipo de hombre se quiere formar?; en el ámbito curricular, ¿a través de qué contenidos y experiencias?; en la planeación académica, ¿qué didácticas utilizará el docente para el desarrollo de competencias?; en la vertiente sociológica, ¿en quién se centra el proceso de aprendizaje, en el docente 0 en el discente?; en la esfera psicológica, ¿ a qué ritmo debe generarse el proceso de aprendizaje?; y en el escenario evaluativo ¿qué concepciones gravitan alrededor de la evaluación integral de cara al desarrollo de competencias?

De los anteriores cuestionamientos es menester resaltar que lo epistemológico se constituyéndose en el eje sobre el cual giran las otras dimensiones, para generar los denominados factores discrepantes del asombro que minimizan la monotonía y la uniformidad en el aula, Dewey (2007).

i. Postura del Modelo Pedagógico Frente al Currículo

En consonancia con el concepto de Vygotsky, (1985) el currículo es la instancia mediadora entre la institución y la sociedad, representa desde el enfoque sistémico una dimensión sociocultural, en donde los actores de la transposición didáctica en los proceso de enseñanza y aprendizaje se encuentra influenciado por la dinámica de contexto; de hecho, Bruner (1988) afirma que el currículo posee una impronta transcendental en el desarrollo humano. En este orden de ideas, el currículo se explica como un resultado dialéctico de la dinámica histórica y cultural, por lo tanto en continua construcción, Carr y Kemmis (1983). Aludiendo a Sacristán Gimeno y Pérez Gómez (1983), se encuentra que el concepto de currículo tiene una connotación de integralidad y flexibilidad que permite planificar, evaluar y justificar un PEP.

Así mismo, parafraseando a Zabalza (1987), el currículo abarca el conjunto de propósitos, competencias cognoscitivas, praxiológicas y actitudinales, es decir, un esquema integral y flexible, provisto de una razón para cada una de ellas. En este sentido, para reflexionar sobre el reto de cambiar, resignificar 0 transformar una arquitectura curricular y así alinearla con el modelo pedagógico, a partir de los códigos que la identifican, es posible identificar ciertas tendencias que se sintetiza a continuación, (Barreto, 2010): El currículo como sustento de la función docencia, (Posner, 2002). El currículo integrado, por competencias y estratégico. El currículo flexible, Pedroza (2005). El currículo transversal (Chueque, Bazán, Lamas, Griffero, Díaz. 1994).

En cuanto a los enfoques curriculares, los proyectos educativos no deben sustraerse de este ámbito, en virtud que guardan total coherencia con las dimensiones del modelo pedagógico. Así, un currículo técnico se inscribe en el paradigma positivista, que de acuerdo a Carr (1990), es la expresión de la pedagogía por objetivos. El práctico, se clasifica en el paradigma fenomenológico, surge como constructo opuesto al enfoque técnico, al respecto, Carr y Kemmis (1988) argumenta el ámbito constructivista del currículo elaborado desde las realidades concretas. El sociocrítico, sustentado en la teoría comunicacional de Habermas y en la escuela crítica alemana de Frankfurt (Arancibia, 2001), según Giroux (1992) maximiza los 
factores de cambio sociales. habría que decir también, acudiendo a Braga (1999), que desde la concepción curricular el interés técnico no es concurrente ni compatible con el práctico y en consecuencia se encuentra en total discrepancia con el crítico.

\section{METODOLOGÍA:}

Se optó por el paradigma cualitativo, con un tipo de estudio hermenéutico en razón que se orienta a comprender y profundizar la realidad de los PEP de las unidades de análisis desde la perspectiva de las dimensiones de los modelos pedagógicos, en un esquema de caracterización autor, texto y contexto, articulando cuidadosamente la semiótica, la semántica y la pragmática en una secuencia de descontextualización, recontextualización para finalizar con una contextualización.

En este orden de situaciones, a partir del análisis los aspectos curriculares de los PEP de los programas académicos: Administración de Empresas, Contaduría Pública, Comercio Internacional, Mercadeo y Economía , y la pregunta problémica ¿cuáles son los lineamientos de modelo pedagógico predominantes en los PEP? se acudió al enfoque histórico hermenéutico, como perspectiva interpretativa, adoptando la línea planteada por Sánchez (2001), en tres etapas: (i) el establecimiento de un conjunto de textos, llamado nivel empírico; (ii) la interpretación de dichos textos; (iii) y la síntesis sobre el primer y tercer punto, es importante aclarar que las dos últimas etapas corresponden al nivel interpretativo en sí.

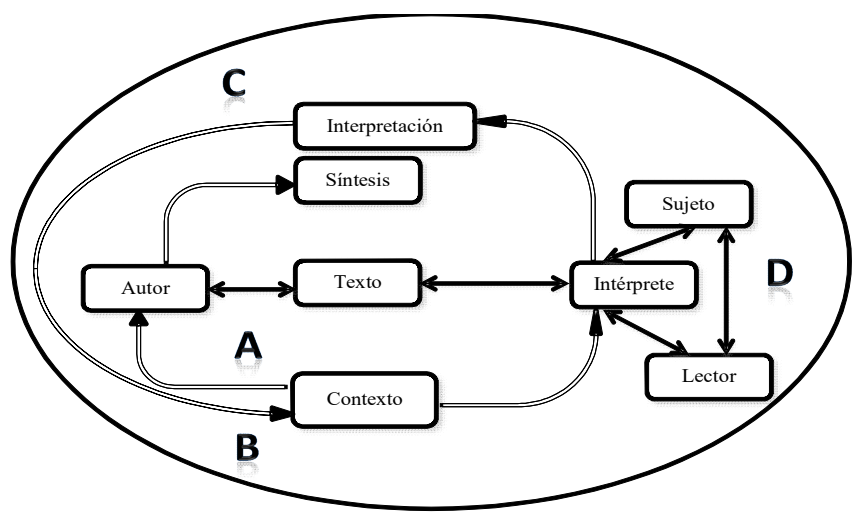

Figura 1. Modelo de Análisis Hermenéutico. Adaptado de Baeza (2002)

Lo escrito hasta aquí supone que los elementos diacrónicos, intencionales o literales no se dieron en términos absolutos, sino que están en función de la situación objetivada en la que intervenga el sujeto cognoscente, con el fin de identificar los rasgos de modelo pedagógico presentes en los Proyectos Educativos de los Programas -PEP- de la Facultad de Ciencias Económicas y Administrativas de la Universidad de Nariño. En consecuencia, se decidió interpretar el conjunto de parámetros que los determinan, analizando las siguientes dimensiones: epistemológica, filosófica, planificación escolar, curricular, sociológica, psicológica y evaluativa, las cuales se las asoció con factores determinantes de los modelos pedagógicos heteroestructurante, autoestructurante e interestructurante.

4. RESULTADOS: LINEAMIENTOS DE MODELO PEDAGÓGICO PRESENTES EN LOS PROYECTOS EDUCATIVOS DE LOS PROGRAMAS.

4.1. Proyectos Educativos del Departamento de Administración y Finanzas

Con respecto a los PEP de Administración de Empresas y Contaduría Pública, se infiere que la corriente crítica y la pedagogía problémica sustentan los alcances del modelo pedagógico. En términos generales, como se puede observar en el Gráfico 1, en la evaluación de la dimensiones correspondientes, permiten visualizar que tiene un fuerte sesgo constructivista, seguido de manera preponderante por una marcado espacio interestructurante, dejando rezagada ciertas trazas tradicionales y conductistas.

En cambio en el programa de Contaduría Pública, perteneciendo a la misma unidad académica, en el PEP se expresa que el modelo pedagógico es problematizador; al respecto, según De Zubiría (2003), dicha alternativa no se la puede catalogar como modelo sino como didáctica, la cual es clasificado por el autor como contemporánea dentro del grupo de la estructural cognitiva y el $A B P$, en las didácticas funcionales. Al margen de la anterior observación, se identificó un fuerte sesgo heteroestructurante, con lineamientos autoestructurantes, particularmente en lo que respecta a la dimensión epistemológica, filosófica y evaluativa, aunque se avizora que existe un asomo de un espacio interestructurante, que seguramente ha venido evolucionando a la par del acontecer del programa académico.

Los resultados se pueden contrastar en la figura $\mathrm{N}^{\circ} 2$.

Figura 2. Lineamientos de modelo pedagógico identificados en los programas académicos del Departamento de Administración y Finanzas de la Universidad de Nariño. 


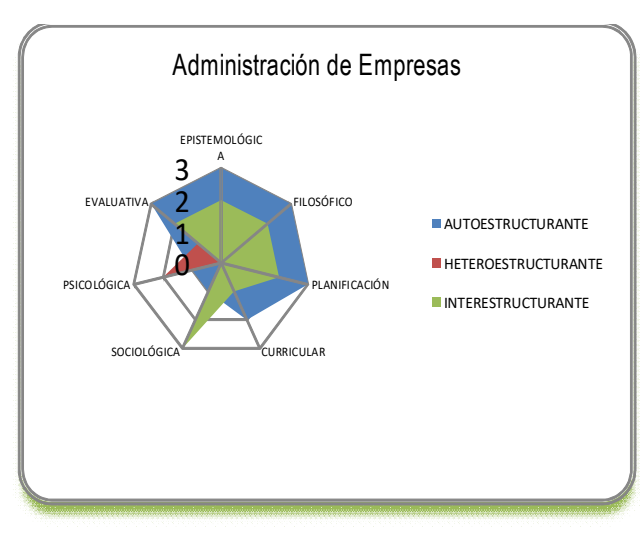

Fuente: Elaboración Propia. Software Excel.

Se observa que los programas académicos tienen un punto de encuentro en la dimisión epistemológica y filosófica, toda vez que se avizora un sesgo orientado al pensamiento crítico y al reto de la complejidad. Se colige que la orientación teórica de los programas está suficientemente argumentada y permite categorizar la visión del sujeto y objeto de transformación. No obstante se identifica una serie de discrepancias en el resto de dimensiones.

Desde la planificación, el PEP de Administración de Empresas es congruente con las bases teóricas que sustentan a la pedagogía problémica. Se infiere la presencia de un modelo didáctico concurrente con las pedagogías activas, que se van diluyendo en la dimensión curricular, en donde se observa un enfoque con características tanto técnicas como prácticas, aunque esta mixtura puede resultar en confrontación, en razón que el enfoque práctico se clasifica en el paradigma fenomenológico y surge como constructo opuesto al enfoque técnico. Para Contaduría se concluye que el programa se diversifica en una postura plurimetodológico, en cuya estructura curricular se observa una pedagogía por objetivos; tal vez por sus característica disciplinar es posible deducir que en ciertos espacios académicos simplifica los preceptos educativos a ciencia aplicada, de ahí que es posible avizorar que dentro de la convergencia teórica en la que se sustenta se encuentran trazas del paradigma positivista y la psicología conductista.

En cuanto a la dimensión sociológica, se evidencian una marcada diferencia, dado que el programa de Administración de Empresas focaliza el proceso de aprendizaje es el estudiante, en tanto que en el de Contaduría Pública sobresalen iniciativas que aluden a la reproducción del saber; seguramente, el contexto disciplinar del programa exhorta a que predomine la escuela transmisionista, en donde la acción y el rol del docente es magistrocentrico, cuya característica

Fundamental se rige por los contenidos, conceptos y saberes. Complementariamente, en el campo psicológico, en los dos PEP se hacen comentarios tenues sobre la pluralidad de individuos, cada uno con un estilo cognitivo, afectivo y vincular; con un cúmulo de expectativas diferentes y hasta opuestas entre sí, que en esencia no permite identificar la respuesta sobre el ritmo en el que debe generarse el aprendizaje.

En términos de evaluación, se esgrime la evaluación integral del estudiante, empero, no se explica cómo se monitoreará el desarrollo de las competencias definidas; en ciertos espacios se configura como simple instrumento de promoción o permanencia.

\subsection{Proyectos Educativos del Departamento de Comercio} Internacional y Mercadeo

En los PEP de los dos programas, al igual que el de Contaduría Pública, se reduce el modelo pedagógico a una didáctica problematizadora. No obstante, a partir del ejercicio hermenéutico, se identifica que sobresalen los lineamientos de un modelo pedagógico autoestructurante, con una fuerte tendencia interestructurante, particularmente en las dimensiones psicológica y filosófica. De otra parte, aunque con un espacio reducido, se encontró que desde la evaluación y lo epistemológica persisten un espacio heteroestructurante. 
Figura 3. Lineamientos de modelo pedagógico identificados en el programa de economía.
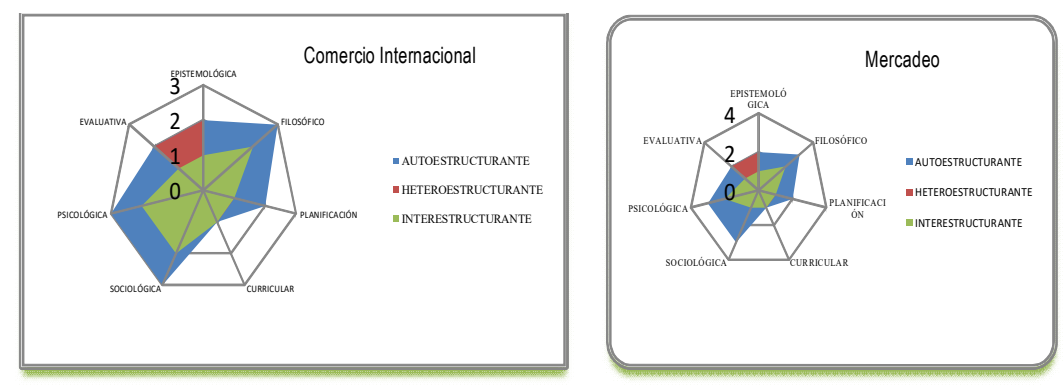

Fuente: Elaboración Propia. Software Excel

En la dimensión epistemológica, se sustentan en el positivismo, en el paradigma sociocrítico y en la hermenéutica. Al respecto, VigotsKy (1991) plantea que los intentos eclécticos de sincronizar elementos heterogéneos, de distinta naturaleza y origen científico se alejan del precepto de la sistematicidad y genera una irrupción brusca de sus bondades que se asumen dispuestas en favor del acto educativo. La anterior postura es eventualmente cuestionable debido a la dificultad de integrar y complementar el paradigma positivista centrado en lo cuantitativo hasta el polo opuesto del constructivismo, en una mixtura que recorre todas las gamas epistemológicas. En la esfera filosófica se focalizan argumentos del materialismo dialéctico, de cuya postura se infiere que pretende explicar desde esta postura los fenómenos económicos, políticos y sociales de la globalización, con lo cual posibilita que se la asuma de manera glocal e integradora, favoreciendo el pensamiento crítico y la sensibilidad social, liberando las mentes de los educandos. Además, es pertinente interpretar que dicha postura filosófica se presenta como un espacio conciliador para que un escenario tan propio del neoliberalismo como lo es el mercadeo, especialmente desde la perspectiva consumista, genere un espacio de reflexión para evitar estar bajo el dominio de prácticas potencialmente alienadoras sin responsabilidad social empresarial. En la dimensión planificación, como se había anunciado, se enarbola la pedagogía problémica, la cual resulta ventajosa en el contexto del aprendizaje significativo y el pensamiento crítico, por ende, en el desarrollo de competencias profesionales. En lo concerniente al plano curricular, se observó elementos predominantes del currículo técnico, identificado por la premisa esbozada entorno a la interacción de contenidos de los cursos, de cuya secuencia se puede deducir que llevado a la práctica en el aula el aprendizaje es concebido como adquisición, retención, acumulación y reproducción de información. En el espacio sociológico, se plantea que el estudiante ocupa el lugar central todo el proceso gravita alrededor de su aprendizaje. En la dimensión psicológica, en el PEP se presenta una propuesta comprensiva del universitario como un actor dinámico en los procesos de enseñanza y aprendizaje; además, expresa el compromiso que al respecto debe tener el docente de cara a los retos cognoscitivos y afectivos. En el escenario evaluativo, se encontró que la focalizan en el seguimiento de la autogestión formativa y se constituye en la guía del proceso pedagógico. Sin embargo no se puede omitir que también la asumen como un instrumento para calificar objetivos de las llamadas "asignaturas", sesgándose en los momentos finales del proceso, desdibujando los propósitos de evaluación mencionados.

En general se puede evidenciar que en la Unidad Académica en análisis se tiene un concepto más unificado del PEP, con el fin de implementar con cierta convergencia el modelo pedagógico. Es claro que predomina la autoestructuración, particularmente en el programa de Mercadeo.

4.3. Proyecto Educativo del Programa de Economía El programa de Economía plantea en su PEP que no se orienta por un solo paradigma, es más, se puede inferir que también transita por una vertiente ecléctica, con rasgos que permiten deducir que predominan lineamientos pedagógicos autoestructurantes, con una fuerte tendencia interestructurante, como se esquematiza en la figura 4. En este sentido, es importante registrar que es el único programa de la Facultad en el que no se encontró trazas conductistas 0 de la pedagogía tradicional, en lo que al PEP concierne. 
Figura 4. Lineamientos de modelo pedagógico identificados en el programa de Economía

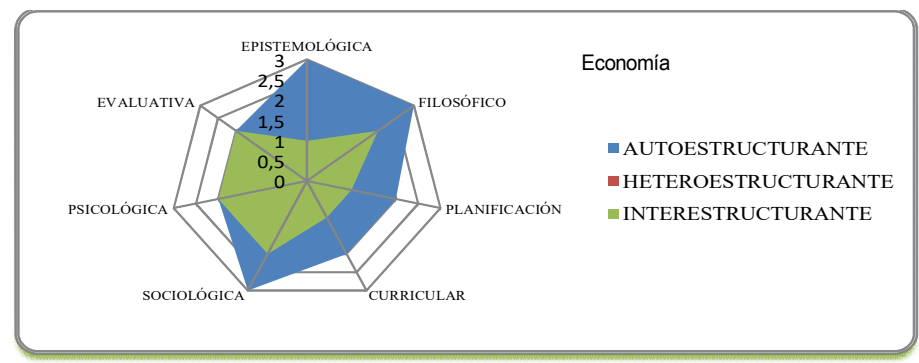

fuente: Elaboración Propia. Software Excel..

La dimensión epistemológica, es posible interpretar que se procura alejarse de los constructos que tornan abstracta la enseñanza y se plantea un enfoque multiparadigmático en el que se analiza en contexto las corrientes tradicionalmente dominantes, del tal forma que favorece reflexionar al discente, con un realismo pragmático, la misión y visión de la economía, propicio para el desarrollo del pensamiento crítico. Desde la argumentación filosófica, se concentran en constructos que respaldan la economía del desarrollo y la corriente crítica. En este sentido, se encuentran planteamientos que coinciden con su postura paradigmática de implementar procesos de enseñanza y aprendizaje a partir del pluralismo teórico, para apropiar en el estudiante diferentes visiones del mundo, generando un análisis crítico en cual se colige será traducido en las funciones sustantivas. En relación a la planificación, guardando cierta similitud con el programa de Contaduría Pública; se infiere que el programa adopta una posición ecléctica, lo cual es consecuente con su fundamentación epistemológica y filosófica, aunque la tarea determinante es valorar las posibles contradicciones implícitos en los diferentes teorías, lo cual no quiere decir que sea prudente subyugarse tímidamente a los preceptos de una teoría dominante, explicado por la crisis paradigmática. En lo concerniente a la dimensión curricular se propone un enfoque emancipador, crítico, problémico y dialéctico por excelencia, de ahí que se infiere que las funciones sustantivas se disponen a resolver los problemas de la realidad del entorno, por tal motivo asume que la fuente natural de los contenidos deben ser las condiciones del contexto; es decir, la praxis en la que se orienta el programa de Economía gravita entre la reflexión y la acción. En lo sociológico, se identificó que el centro del proceso es el estudiante, quien construye su propio conocimiento mediante el diálogo de lo pensado, para poder debatir sus ideas. En la dimensión psicológica, no se establece de manera expresa tiempos relacionados con el ritmo de aprendizaje. Aunque se puede deducir que no se ha soslayado ese propósito, toda vez que se hace referencia en mitigar las potenciales limitantes representada en la heterogeneidad en los ritmos de aprendizaje a través de las oportunidades que brinda la pedagogía dialogante que en el PEP se manifiesta. Se menciona que la evaluación correspondiente a la pedagogía tradicional está desapareciendo, dándole paso a la integralidad de las dimensiones humanas propias de la interestructuración. En este sentido, la evaluación tiene un propósito de conceder al estudiante la oportunidad de seguir aprendiendo de las experiencias significativas de sus pares y de la que en él acontece. Sin embargo, se puede apreciar huellas conductistas en el esquema de evaluación que presente en el estatuto estudiantil de la Universidad.

En general, los resultados invitan a pensar si se privilegia el eclecticismo o simplemente se adopta opciones pedagógicas dispersas, ver figura 5 .

\section{Figura 5. Relación de los Modelos Pedagógicos con los} PEP.

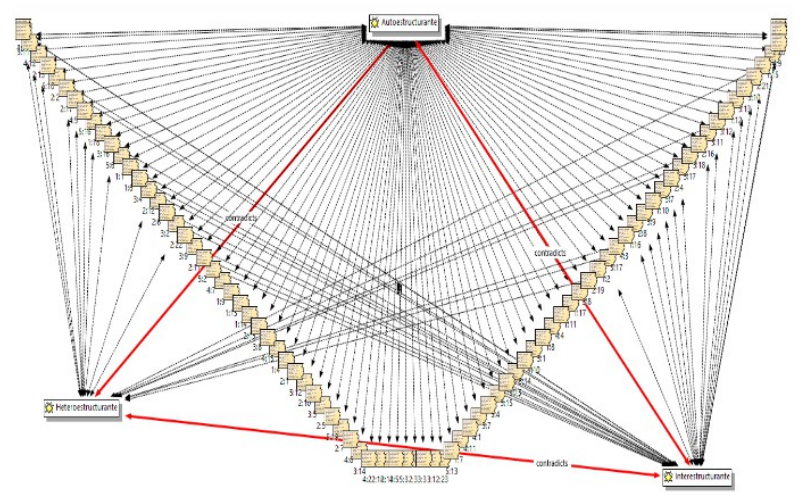

Fuente: Elaboración Propia, Software Atlas Ti. 


\section{DISCUSIÓN:}

La estructuración de un PEP no puede limitarse al cumplimiento de la normatividad que reglamenta el registro calificado, como lo es en la actualidad el Decreto 1075 de 2015, sino que debe asociarse con el alcance integral del modelo pedagógico, el cual debe ser planeado, apropiado e implementado como es el medio fundamental del PEI y su evaluación debe estar en función del desarrollo de las funciones sustantivas.

Los estructuradores del PEP, esencialmente, deben dominar el metalenguaje pertinente, para que actuando en consecuencia puedan establecer la diferencia entre modelo educativo y modelo pedagógico y las consideraciones sobre el propósito de un proyecto educativo. Para una adecuada construcción teórica de un proyecto educativo, seguir la ruta de las dimensiones del modelo pedagógico es una opción procedente

Lo escrito hasta aquí supone que los modelos pedagógicos buscan en la filosofía de la educación respuestas a cuestionamientos relacionados con la complejidad del ser humano (Díaz y Quiroz. 2001), en una tarea cuya esencia es armonizar el proceso educativo, en el sentido de determinar qué enseñar, a quiénes y con qué procedimiento (Flórez, 2005). Por consiguiente, en un PEP se deberá indicar como se asume lo pedagógico en un programa académico, evitando que se limite a una instancia enunciativa, de manera que resulta un ejercicio de reflexión colectiva de la realidad en la que habita la Universidad y sus programas académicos.

Al mismo tiempo, es imperativo reflexionar sobre el proceso evaluativo, el cual debe trascender el criterio discriminatorio de promoción o permanencia, concibiendo una información amplia de las dimensiones humanas del estudiante (Rojas y Ospina, 2011).

Estructurar un modelo pedagógico en un PEP tienen implicaciones curriculares, que deben ser asumidos con rigor por los actores del proceso, especialmente en la transposición didáctica, que no es más que la transformación del saber experto en saber enseñado Chevallard (1985).

De acuerdo a la pesquisa documental relacionada con el estudio, en los PEP no se encontró de manera que los programas académicos se acojan a un modelo pedagógico determinado, más aún, los hallazgos sugieren la existencia de un eclecticismo, sin embargo, no se encuentra un plan de acción que permita al profesor apropiarse de las posturas pedagógicas relacionadas, que eviten los
Pseudoposicionamientos proclives a la tergiversación y a la ambigüedad.

Siguiendo los postulados de Claret (2003), en los PEP se debe plantear esquemas que permitan reflexionar, reproducir, apropiar y aplicar los diferentes espacios epistemológicos y al mismo tiempo integrar los preconceptos del discente con los conceptos del profesor en un encuentro pedagógico participativo y equilibrado, con una evaluación por procesos, permanente e integral.

Con una visión global, llama la atención que de cara al advenimiento de la llama cuarta revolución industrial, que no se realice una propuesta interestructurante y dialogante, relacionada con la innovación tecnológica, que favorezca la creación de formas de aprendizaje innovadoras, centradas en el que aprende y el que enseña.

Es importante consignar que todos los programas académicos analizados reconocen que el conocimiento es una construcción externa al aula y que la reiteración cumple una función determinante en la transposición didáctica; a pesar de que los aspectos aludidos suelen ser negados por los modelos autoestructurantes, que son los rasgos predominantes en ellos. Es menester reflexionar que no se ahonda en los cursos alternativos de acción pedagógicos que permitan el encuentro del conocimiento que se construye por fuera del aula y el que debe ser reconstruido de manera activa e interestructurada, a través del diálogo suscitado entre el estudiante, el saber y el docente.

Indiscutiblemente, lo más relevante identificado en los PEP es la identificación de los profesores como actores estratégicos de enlace frente a la dimensión cognitiva de los discentes, con el propósito de trascender la tarea de transmitir conocimientos, focalizándose en desarrollar en el estudiante su nivel cognitivo, praxiológico y actitudinal, que provienen de la interestructuración entre el sujeto y el entorno sociocultural determinado.

Por otra parte, persisten trazas heteroestructurantes, cuya limitante potencial es la reducción de la finalidad educativa a la acción "repetición y corrección", por parte del docente y de la "imitación y copia" por parte del estudiante, las cuales deben ser rápidamente superadas porque se oponen al pensamiento crítico y al constructivismo.

La variedad de posturas pedagógicas asumidas por los programas académicos de la FACEA, permiten deducir la validez de la implementación de un enfoque plurimetodológico. Sin embargo, como lo afirma Gagné (1985), el adjudicarse estar en una escuela seleccionadora, exige estar racionalmente organizado y evidenciar 
sistematicidad, con el fin de evitar asumir en la función docencia arbitrariedades pedagógicas, en virtud que adoptar una posición ecléctica enfrenta al docente, como responsable de los lineamientos pedagógicos y de estrategias didácticas a apropiarse de los siguientes principios: selección, secuencia, pertinencia y coherencia. (Salazar \& Bastidas, 2005).

Definitivamente, acoger un enfoque ecléctico implica un especial compromiso con las diferentes dimensiones de competencias para que los discentes logren transferir los aprendizajes a contextos reales. El sesgo constructivista que habita en el enfoque plurimetodológico de los PEP, hace más compleja la tarea de poner en escena esta alternativa pedagógica, especialmente si se relaciona con el denominado aprendizaje significativo, que según Novak y Gowin (1988), es la contraposición de los postulados inherentes en el modelo autoestructurante.

\section{CONCLUSIONES:}

En los documentos analizados no se evidencia una conceptualización clara del alcance del modelo pedagógico, situación susceptible de considerarse una debilidad mayor, en virtud que es el PEP en el medio fundamental del PEI y su adecuada estructuración e implementación se correlacional con el desarrollo de las funciones sustantivas. En los PEP analizados, el epígrafe "modelo pedagógico", se lo asocia con la enseñanza problémica, la cual en sí es una alternativa didáctica.

Desde las dimensiones del modelo pedagógico, predomina un esquema autoestructurante; se acoge aportes de los diversos constructivismos enriquecidos a partir de la teoría sociocrítica, que posibilitan el análisis, el desarrollo y le otorgan un matiz de innovación a los procesos de enseñanza y aprendizaje, en donde se enfatiza en el sujeto de transformación, encaminado a encontrar sentido a la interacción educativa en la que participa; no obstante, no se identificó respuestas claras frente al advenimiento de la cuarta revolución industrial, como realidad de contexto.

Desde la dimensión filosófica, psicológica y sociológica, se infiere que los programas académicos analizados se están alejando de los modelos transmisionista unidireccionales, aunque no se ha superado las estructuras curriculares cerradas, en donde prevalecen aún los contenidos y los esquemas didácticos basados en la secuenciación.

Los lineamientos identificados guardan coherencia con el desarrollo individual y con los principales problemas de la región de su área de influencia, haciendo de la interacción universitaria un escenario más dialéctico que dialogal, propendiendo que el discente adquiera un pensamiento crítico, creativo de la realidad en la que habita.
Otro rasgo, aunque menos relevante que el autoestructurante, son los de connotación interestructurante, que se presentan como alternativa pertinente en la configuración de una síntesis dialógica. Todos los programas académicos analizados reconocen que el conocimiento es una construcción externa al aula y que la repetición cumple una función determinante en la transposición didáctica, aunque no se profundiza en las estrategias que permiten su reconstrucción de manera activa e interestructurada, a través del diálogo suscitado entre el estudiante, el saber y el docente.

Indiscutiblemente, lo más relevante que se logró identificar en la documentación analizada, se resume en el hecho de que los profesores se constituyen en los actores estratégicos de enlace frente a la dimensión cognitiva de los discentes, con el propósito de trascender la tarea de transmitir conocimientos, focalizándose en un ámbito desarrollista.

Así las cosas, se infiere que se está adoptando un currículo de transición, toda vez que el conductismo es progresivamente reemplazado por metodologías integradoras de carácter holístico, que incorporan una pedagogía afectiva y cambio axiológico en el marco de un sesgo constructivista.

Con una visión global, se identificó que los lineamientos más atenuados en los PEP, con excepción del programa de Contaduría Pública, son los que identifican al modelo pedagógico heteroestructurante, cuya limitante potencial es la reducción de la finalidad educativa a la acción "repetición y corrección", por parte del docente y de la "imitación y copia" por parte del estudiante.

En general, en las estructuras curriculares se observa un peso excesivo destinado a los contenidos y a las informaciones específicas, que normalmente limitan los espacios heurísticos. La variedad de posturas pedagógicas asumidas por los programas académicos de la FACEA, permiten colegir la validez de la implementación de un enfoque plurimetodológico en los procesos de enseñanza y aprendizaje, el cual es una tendencia educativa valida con la complejidad en la que habita la Universidad del siglo $\mathrm{XXI}$, no obstante su implementación es más compleja porque implica buscar un punto de encuentro a una serie de contradicciones de connotación epistemológica. 


\section{REFERENCIAS:}

Arancibia, M. (2001). Reflexiones en torno a la aplicabilidad pedagógica de la informática: apuntes para un trabajo transdisciplinario en el currículo escolar. Estudios pedagógicos (Valdivia), (27), 75-95. Recuperado de: http://www.scielo.cl/scielo.php? script=sci_arttext\&pid=S0718$07052001000100006 \&$ Ing $=e n \&$ tlng=es. $10.4067 /$ S0718-07052001000100006.

Baeza, M. (2002). De las metodologías cualitativas en investigación científico social. Diseño y uso de instrumentos en la producción de sentido " Concepción: Editorial de la Universidad de Concepción.

Barreto, N. (2010).Tendencias curriculares a considerar en los procesos de cambio educacional. Quaderns Digitales No. 16. Recuperado de: http://www.quadernsdigitals.net/index.php? accionMenu=hemeroteca.VisualizaArticulolU.visu aliza\&articulo_id=10905

Braga G. (1999): Diseño, desarrollo e innovación del currículum. (Proyecto Docente)

Bruner, J. (1988). Desarrollo cognitivo y educación. Madrid: Morata.

Canfux, V. (1996). "Tendencias Pedagógicas Contemporáneas". CEPES. La Habana.

Carr, W. (1990). Hacia una ciencia crítica de la educación. Hacia una investigación educativa crítica. Barcelona: Laertes.

Carr, W. y Kemmis, S. (1988). Teoría crítica de la enseñanza. Barcelona: Ediciones Martínez Roca.

(1983). Becoming critical. Krowing throug Action Research. Deakin University, Victoria.

Cardoso, H. (2007). Del proyecto educativo al modelo pedagógico. Odiseo, revista electrónica de pedagogía, 4(8). Recuperado en:
Chevallard Y. (1985) La transposition didactique ; du savoir savant au savoir enseigné, Paris, La Pensée Sauvage.

Chueque, M, Bazán, I, Lamas M, Griffero M, Díaz P. (1994). La inter y transdisciplinariedad: una cuestión pendiente. Docentes Cátedra Administración Unidades de InformaciónFacultad de Humanidades- Universidad Nacional de Mar del Plata. Argentina.

Claret. (2003). Pedagogía del Conocimiento. Segunda Edición. Ed: Mc Graw Hill. Bogotá.

De Zubiría, J. (2011) Los Modelos Pedagógicos, Hacia Una Pedagogía Dialogante. Bogotá. Cooperativa Editorial Magisterio, Tercera Edición.

De Zubiría, M. (2003). Enfoques Pedagógicos y Didácticas Contemporáneas. Fundación Internacional de Pedagogía Conceptual. Copyright. Bogotá.

Dewey, J. (2007). Cómo pensamos. La relación entre pensamiento reflexivo y proceso educativo. Barcelona: Paidós

Díaz, A. y Quiroz, R. (2001). Corrientes pedagógicas, modelos pedagógicos y enfoques curriculares. Relación sistemática entre ellos. Revista avanzada, 10, 116 - 129

Falik, L. y Feuerstein, R. (1990). Modificabilidad de la Estructura Cognitiva: Una Nueva Perspectiva para el asesoramiento y la psicoterapia. International Journal of Cognitive Educación y Aprendizaje Mediado.

Flórez, R. (1994). Hacia una pedagogía del conocimiento. Santafé de Bogotá: McGraw-Hill.

. (2005). Pedagogía del Conocimiento. Colombia. McGraw-Hill Interamericana, S.A.

Feuerstein, R. \& Hoffman, M. (1992). Programa de Enriquecimiento Instrumental. Madrid, España. Bruño.

Gagné, R. (1985). Las condiciones del aprendizaje. 4ta. edición. México: McGraw-Hill.

Giroux, H. (1992). Teoría y resistencia en educación. México D.F.: Ediciones Siglo XXI

Joyce, B., Weil, M. y Calhoun, E. (2002). Modelos de enseñanza. Barcelona, España: Gedisa

Kuhn, T. (1986). La estructura de las revoluciones científicas. México: Breviarios.

Medina, A. Mata, F. S., \& Arroyo, R. (2002). Didáctica general. Madrid: Prentice Hall. 
Morales, G. (2008). Interacciones e Implicaciones Entre Filosofía Y Educación. Sophia, Colección de Filosofía de la Educación, núm. 4, Universidad Politécnica Salesiana Cuenca, Ecuador. Recuperado de: http://www.redalyc.org/pdf/4418/4418 46110003.pdf

Morín, E. (2003). Los siete saberes necesarios para una educación de futuro. Barcelona: Paidós.

NOT, L. (1983). Las pedagogías del conocimiento. México: Fondo de cultura Económica. 1ra. edición en español.

(1992). La enseñanza dialogante. Madrid: Herder

Novak, J. y Gowin, D. (1988). Aprendiendo a aprender. Barcelona: Martínez Roca.

Pedroza, R. (2005) "La flexibilidad académica en la universidad pública", pp. 19-41, en Flexibilidad académica y curricular en las instituciones de educación superior, René Pedroza Flores, Bernardino García Briceño, comps. México : M.A., Porrúa.

Posner, G. (2002) Análisis del curriculo. Bogotá: Mc Graw Hill.

Rojas, W; Ospina, C. (2011). Consideraciones sobre el sentido de un proyecto educativo en Contaduría Pública. Cuadernos de Administración. Revistas de Administración y Negocios. Vol. 27. No. 45. Recuperado

de: http://cuadernosdeadministracion.univa lle.edu.co/index.php/cuadernosadmin/a rticle/view/616/2490

Sacristán, G. Pérez, G. (1983). La Enseñanza: su teoría y su práctica. Akal. Madrid.

Sánchez, A. (2001). El método hermenéutico aplicado a un nuevo Canon: hacia la autorización de la producción escrita de los estudiantes de inglés. CAUCE, Revista de Filología y su Didáctica.

Salazar, L, \& Batista, J. (2005). Hacia la consolidación de un enfoque ecléctico en la enseñanza de idiomas extranjeros. Paradígma, 26(1), 55-88. Recuperado de: http://www.scielo.org.ve/scielo.php? script=sci_arttext\&pid=S1011$22512005000100004 \&$ Ing=es\&tlng=es
Vigotsky, L. (1985). Pensamiento y lenguaje, Pléyade. Buenos Aires. España.

. (1991). Obras Escogidas. Tomo I. Visor.

Zábálzá, M. A. (1987): «Diseño y desarrollo curricular». Narcea, Madrid. 